\title{
Ação de fungicidas sobre o crescimento do fungo entomopatogênico Metarhizium sp.
}

\author{
Action of fungicides on growth of Metarhizium sp.
}

Silvana Damin ${ }^{[a]}$, Andréia Vilani[a], Danusa de Freitas ${ }^{[a]}$, Cristiane Krasburg ${ }^{[a]}$, Jéssica Alves de Queiroz ${ }^{[a]}$, Francini Yumi Kagimura ${ }^{[b]}$, Sideney Becker Onofre ${ }^{[c]}$

[a] Bolsistas PIBIC/UNIPAR, biólogas, Graduadas pela Universidade Paranaense (UNIPAR), Francisco Beltrão, PR - Brasil, e-mail: sil_damin@hotmail.com

[b] Bolsista PIBIC/UNIPAR, acadêmica de Biomedicina da Universidade Paranaense (UNIPAR), Francisco Beltrão, PR - Brasil, e-mail: fran_yk@hotmail.com

[c] Biólogo, Doutor em Processos Biotecnológicos, professor titular da Universidade Paranaense (UNIPAR), Francisco Beltrão, PR - Brasil, e-mail: sideney@unipar.br

\section{Resumo}

O uso abusivo de inseticidas químicos na agricultura pode trazer grandes prejuízos à saúde humana, ao meio ambiente e pode causar o aumento na abundância de pragas agrícolas, graças ao desequilíbrio resultante da destruição de inimigos naturais, como fungos entomopatogênicos, importantes no controle biológico de pragas agrícolas. 0 trabalho visa a avaliar a interferência de dois fungicidas químicos, $\mathrm{Nativo}^{\circledR} \mathrm{e} \mathrm{Bendazol}^{\circledR}$, sob o desenvolvimento dos fungos Metarhizium anisopliae var. acridum; Metarhizium flavoviride var. flavoviride; Metarhizium anisopliae var. lepidiotum, sendo utilizados no controle biológico na cultura da soja. Os testes foram realizados in vitro, em meio de cultura BDA, na presença dos fungicidas na concentração recomendada para uso no campo em seis diluições e teste controle. Foi avaliado o efeito tóxico do fungicida por meio do crescimento vegetativo do fungo. $O$ fungicida Nativo ${ }^{\circledR}$ apresentou CIM de $20 \mu \mathrm{L} / \mathrm{L}$, para o fungo $M$. anisopliae var. acridum; CIM de $150 \mu \mathrm{L} / \mathrm{L}$ para o fungo $M$. flavoviride var. flavoviride; CIM de $350 \mu \mathrm{L} / \mathrm{L}$, para o fungo M. anisopliae var. lepidiotum. 0 fungicida Bendazol ${ }^{\circledR}$ apresentou CIM de $170 \mu \mathrm{L} / \mathrm{L}$ para o fungo $M$. anisopliae var. acridum; CIM de $350 \mu \mathrm{L} / \mathrm{L}$, para o fungo $M$. flavoviride var. flavoviride; CIM de $350 \mu \mathrm{L} / \mathrm{L}$, para o fungo M. anisopliae var. lepidiotum. Dessa forma, pode-se concluir que os fungicidas Nativo ${ }^{\circledR} \mathrm{e} \mathrm{Bendazol}^{\circledR}$ inibem o crescimento das linhagens de Metarhizium sp.

Palavras-chave: Controle biológico. Inibidores. Entomopatogênicos. Pragas agrícolas.

\section{Abstract}

The overuse of chemical pesticides in agriculture can bring great prejudice to human health, to the environment, and may cause an increase in the abundance of agricultural pests due to the unbalance resulting from the destruction of natural enemies such as entomopathogenic fungus. They are very important in biological control 
of the agricultural pests. The study aims to evaluate the interference of two chemical fungicides, Nativo ${ }^{\text {Tm }}$ and Bendazol ${ }^{T M}$ and the development of the fungus Metarhizium anisopliae var. acridum; Metarhizium flavoviride var. flavoviride; Metarhizium anisopliae var. lepidiotum, fungus of interest in biological control of soybean. The tests were performed in vitro with the PDA culture medium in the presence of fungicides in the field concentration, other six dilutions and control test. We evaluated the toxic effect of the fungicide through the fungus' vegetative growth. The fungicide Nativo ${ }^{T M}$ presented (MIC) of $20 \mathrm{~mL} / \mathrm{L}$, for the fungus $\mathrm{M}$. anisopliae var. acridum; MIC of $150 \mathrm{~mL} / \mathrm{L}$ for the fungus M. flavoviride var. flavoviride; MIC of $350 \mathrm{~mL} / \mathrm{L}$ for the fungus $\mathrm{M}$. anisopliae var. lepidiotum. The fungicide Bendazol ${ }^{T M}$ presented (MIC) of $170 \mathrm{~mL} / \mathrm{L}$ for the fungus $\mathrm{M}$. anisopliae var. acridum; MIC of $350 \mathrm{~mL} / \mathrm{L}$. for the fungus M. flavoviride var. flavoviride; MIC of $350 \mathrm{~mL} / \mathrm{L}$. for the fungus M. anisopliae var. lepidiotum. Thus we can conclude that the Nativo ${ }^{T M}$ and Bendazol ${ }^{T M}$, inhibit the growth of strains of Metarhizium sp.

Keywords: Biological control. Inhibitors. Entomopathogenic. Agricultural pests.

\section{Introdução}

A crescente preocupação com o meio ambiente tem ressaltado a importância das pesquisas científicas que procuram diminuir a agressão constante que o ecossistema vem sofrendo por intervenção do próprio homem. Por esse motivo e por outros, como $o$ alto custo dos defensivos e o aumento de resistência das pragas a esses produtos existentes, o número de pesquisas envolvendo organismos capazes de promover o controle biológico de pragas agrícolas e de interesse na saúde publica vem aumentando (DESTÉFANO, 2003).

Essa conscientização ambiental tem resultado na busca, pelo setor agrícola de tecnologias, por implantação de sistemas de produção de enfoque ecológico rentáveis e socialmente justos. Como resposta a essa demanda, buscam-se avanços no desenvolvimento de soluções tecnológicas para uma agricultura sustentável, em que se consiga também controlar fungos, bactérias, insetos, nematoides e vírus, que normalmente são fatores limitantes para a obtenção de altos rendimentos na produção de muitas culturas agrícolas, inclusive a da soja.

Durante todo o seu ciclo, a cultura da soja está sujeita ao ataque de diversas pragas e, segundo Maldaner (2009), no Sul do País, entre as que mais têm preocupado os produtores estão o tamanduá da soja (Sternechus subsignatus), os corós (Demodema brevitarsis, Diloboderus abderus e Phyllophaga triticopha$g a$ ), as vaquinhas (Diabrotica speciosa e outras espécies), a lagarta-elasmo (Elasmopalpus lignosellus), as lesmas e os caracóis, sendo a maioria dessas pragas controladas naturalmente pelo fungo entomopatogênico Metrahizium anisopliae.

A agricultura sustentável, produtiva e ambientalmente equilibrada, apoia-se em práticas agropecuárias que promovam a agrobiodiversidade e os processos biológicos naturais, baseando-se no baixo uso de insumos químicos externos. Infere-se daí que o controle biológico é uma alternativa promissora para o manejo de pragas em sistemas agrícolas sustentáveis, tendo em vista que se constitui em um processo natural de regulação do número de indivíduos da população de pragas por ação dos agentes de mortalidade biótica, os quais são também denominados de inimigos naturais ou agentes de controle biológico (MENEZES, 2006).

Controle biológico é um fenômeno que acontece naturalmente na natureza e que consiste na regulação do número de plantas e animais por inimigos naturais. É uma estratégia que o homem há muito tempo vem utilizando para o controle de patógenos, pragas e ervas daninhas, por meio de inimigos naturais - ação considerada uma arte por muitos cientistas, embora vários esforços tenham sido feitos para transferir o controle biológico para o domínio da ciência (ALVES, 1998b).

Segundo Alves (1998a), o controle biológico possui muitas vantagens: especificidade seletividade; multiplicação, dispersão e produção por meio dos indivíduos da população; efeitos secundários, podendo afetar as gerações seguintes; controle mais duradouro; controle associado a inseticidas; podem ser modificados geneticamente dando origens a linhagens específicas; não poluem e não são tóxicos ao 
meio ambiente; a comercialização de produtos biológicos possibilita a venda mais lucrativa; não ocorre a ressurgência de pragas e, normalmente, os insetos não se tornam resistentes ao controle biológico.

Com mais frequência o controle biológico, por meio de fungos entomopatogênicos, está ganhando espaço no setor agrícola do País. Dentre os fungos entomopatogênicos, destaca-se o gênero Metarhizium sp., que é formado por diversas espécies e variedades.

O fungo M. anisopliae é um fungo cosmopolita e, sem dúvida, é um dos fungos mais estudados em programas de manejo de pragas, uma vez que representa grande potencialidade como entomopatógeno (DESTÉFANO, 2003; ONOFRE et al., 2002). Esse fungo apresenta patogenicidade para mais de 204 espécies de insetos pertencentes a 43 famílias dentre as ordens Orthoptera, Dermaptera, Hemiptera, Lepidoptera, Diptera, Hymenoptera, Coleoptera, Homoptera e outras (DESTÉFANO, 2003).

No Brasil, esse fungo tem sido estudado sobre diversas pragas, como Mahanarva posticata (cigarrinha-da-cana), Diatraea saccharalis (broca-da-cana), Nezara viridula (percevejo-verde da soja), Piezodorus guildini (percevejo-verde-pequeno da soja), Hypothenemus hampei (broca-do-café), apresentando grande potencial no controle biológico. Os insetos atacados tornam-se rígidos e cobertos por uma camada pulverulenta de conídios. No final da conidiogênese, o cadáver pode mostrar tons de verde que variam de claro a escuro, acinzentados ou ainda esbranquiçados com pontos verdes (ALVES, 1998b).

Além de $M$. anisopliae, o $M$. flavoviride também tem sido reconhecido como agente controlador de gafanhotos e outros insetos, já que isolados dessas espécies apresentam virulência elevada, resistência a altas temperaturas e podem, ainda, ser facilmente produzidos em condições laboratoriais (ONOFRE et al., 2002).

Atualmente vem se avaliando experimentalmente o M. anisopliae var. acridum para o controle de carrapatos, mais especificamente sobre B. microplus (ATHAYDE, 2002) com resultados eficientes e estimulantes. Essa espécie pode atacar gafanhotos da espécie Schistocerca pallens, com alta capacidade na produção de proteases, proporcionando a infecção de insetos pelo fungo, estando essas proteases envolvidas na hidrólise dos componentes cuticulares, facilitando a penetração da hifa por meio da cutícula de insetos.
Os fungos entomopatogênicos penetram no hospedeiro via tegumento, o que os coloca em vantagem quando comparados com outros grupos de patógenos, que só entram no inseto por via oral. Evidências obtidas por microscopia eletrônica e histoquímica sugerem que a etapa de penetração ocorre por uma combinação de degradação enzimática e pressão mecânica. Enzimas, como proteases, lípases e quitinases, facilitam a penetração mecânica do fungo e o metabolismo do tubo germinativo. Na área de procutícula, ao redor do local de penetração, aparecem sintomas de histólise (decomposição do tecido por ação enzimática). Algumas enzimas podem estar correlacionadas com a agressividade de certos fungos para determinados hospedeiros, porém, a morte do inseto é ocasionada por uma série de eventos (ALVES, 1998).

A morte do inseto ocorre em média oito dias após o a inoculação dos fungos, por causa da produção de micotoxinas, mudanças patológicas na hemocele, ação histolítica, bloqueio do aparelho digestivo, em razão do crescimento vegetativo e de outros danos físicos, em decorrência do crescimento do micélio e do início do processo de esporulação do fungo e rápida disseminação pelo vento (ALVES, 1998).

Sabe-se que o manejo das pragas agrícolas vem sendo realizado principalmente por meio de agentes químicos, porém, estes podem prejudicar a saúde humana, causar efeitos maléficos em inimigos naturais e polinizadores e gerar poluição no meio ambiente. Em especial, ao considerarmos os fungicidas, podem ser encontrados aspectos negativos em relação aos entomopatógenos, pois estes podem ter a germinação inibida, diminuindo o potencial do inóculo.

Dessa forma, a correta manipulação dos inimigos naturais nos sistemas de produção agrícola e dos produtos químicos com pouca interferência sobre a atividade dos fungos entomopatogênicos é de suma importância para se manter os níveis desses micro-organismos no nível do campo. Dentre os produtos comerciais indicados para o controle de doenças fúngicas da soja que poderiam interferir sobre os fungos entomopatogênicos, destacam-se o Nativo ${ }^{\circledR}$ e o Bendazol ${ }^{\circledR}$.

Segundo a SEAB (2010b), o fungicida Nativo ${ }^{\circledR}$ (trifloxistrobina e tebuconazol) é indicado para o controle de oídio (Erysiphe diffusa), ferrugem asiática (Phakopsora pachyrhizi), crestamento foliar (Cercospora kikuchii), septoriose (Septoria glycines), 
Antracnose (Colletotrichum truncatum) e Mela (Rhizoctonia solani). Já o fungicida Bendazol ${ }^{\circledR}$ (carbendazin) é utilizado no controle de mancha-parda (Septoria glycines), crestamento foliar (Cercospora kikuchii) e oídio. Pertence ao grupo químico dos triazóis e, como tal, inibe a biossíntese do ergosterol e, consequentemente, da membrana celular dos fungos (SEAB, 2010a).

Assim sendo, o presente trabalho teve como objetivo avaliar a toxicidade dos dois fungicidas: $\mathrm{Nativo}^{\circledR}$ e Bendazol ${ }^{\circledR}$, a fim de verificar se estes interferem sobre o crescimento de três linhagens do fungo entomopatogênico Metharizium sp.

\section{Materiais e métodos}

Os ensaios foram conduzidos in vitro, em meio de cultura BDA (batata-dextrose-ágar), adicionando-se os produtos fitossanitários em concentrações preestabelecidas ao meio de cultura fundido ainda não solidificado.

Após a solidificação do meio de cultura contendo o fungicida, foi realizada a inoculação dos micro-organismos por meio de uma alça de platina contendo em sua extremidade conídios das linhagens fúngicas de Metarhizium sp. As placas com culturas puras dos fungos foram incubadas por 15 dias em meio de cultura MC (meio completo) (ALVES et al., 1998a).

As linhagens fúngicas avaliadas foram:

1) Metarhizium anisopliae var. acridum linhagem CG 291, fornecido pelo Departamento de Agricultura dos Estados Unidos (United States Department of Agriculture - Agricultural Research Service - USDA-ARS] [Agricultural Research Entomopathogenic Fungi - ARSEF 324] de propriedade do Australian Commonwealth Scientific and Industrial Research Organisation - CSIRO FI-985, isolado de Austracris guttulosa (Orthoptera: Acrididae) em Queensland, Austrália em 02/1979;

2) Metarhizium flavoviride var. flavoviride linhagem CG 647 fornecido pela CSIRO [FI-1170; ARSEF 2025; Collection Strain Browser - CBS 125.65]. Coletado em 1963 na Alemanha; e

3) Metarhizium anisopliae var. lepidiotum linhagem CG 648 fornecido pela CSIRO [FI-147], isolado de um coleóptero na Austrália em 1974.
Para a realização deste trabalho, inocularam-se as variedades fúngicas no centro de uma placa de Petri, sendo mantidas em câmara climatizada a $28^{\circ} \mathrm{C}$.

A determinação do efeito dos fungicidas sobre o crescimento fúngico foi realizada determinando-se o crescimento vegetativo de cada linhagem, utilizando-se o modelo proposto por Alves Moino e Almeida (1998b), sendo o crescimento vegetativo determinado medindo os diâmetros das colônias em dois sentidos ortogonais na superfície do meio de cultura, considerando-se o seu diâmetro médio.

Para a cultura da soja no controle de doenças fúngicas, foram avaliados dois produtos comerciais recomendados no Brasil. São eles: Nativo ${ }^{\circledR}$, cujos princípios ativos são: trifloxistrobina + tebuconazole, nas concentrações 2,5 mL/L, 1.250, 650, 350, 150, 70 e $40 \mu \mathrm{L} / \mathrm{L}$; e Bendazol ${ }^{\circledR}$, cujo princípio é carbendazin em concentrações de 1,43 mL/L, 750, 350, 170, 85, 43 e $22 \mu \mathrm{L} / \mathrm{L}$. Como controle das três variedades avaliadas foram mantidas em meio BDA na ausência dos fungicidas avaliados.

Todas as avaliações foram realizadas em triplicatas e com os dados foram calculadas as médias, os desvios e a comparação dessas médias pelo teste de Tukey ao nível de 5\% de significância.

\section{Resultados}

Dados obtidos após a observação do crescimento das linhagens CG-291 de Metarhizium anisopliae var. acridum, CG 647 de Metarhizium flavoviride var. flavoviride e da linhagem de CG 648 Metarhizium anisopliae var. lepidiotum em meio BDA, sob condições controladas e ausências de fungicidas encontram-se sumarizados na Tabela 1 e nas Figuras 1, 2 e 3.

Em análise dos dados contidos na Tabela 1, verifica-se que as três linhagens apresentaram resultados semelhantes, quando comparadas entre si, e apresentaram diferenças significativas no decorrer do tempo de observação. 0 resultado observado representa uma característica esperada, tendo em vista que a relação de crescimento das três linhagens foi proporcional ao tempo de observação.

A cinética de crescimento das três linhagens do fungo Metarhizium sp. está apresentada na Figura 2. Com esses dados pode-se perceber que os três fungos tiveram cinética semelhante, em que o fungo 
Tabela 1 - Características de crescimento das três linhagens do fungo Metarhizium em meio BDA, sob condições controladas e ausência de fungicidas - 360 horas de crescimento

\begin{tabular}{lccccc}
\hline & \multicolumn{5}{c}{ Tempo de observação (horas) / halos de crescimento em $\mathbf{~ m m}$} \\
\cline { 2 - 6 } Variedade & $\mathbf{4 8}$ & $\mathbf{1 4 4}$ & 192 & $\mathbf{2 8 8}$ & $\mathbf{3 6 0}$ \\
\hline CG 291 & $5,00 \pm 0,02 \mathrm{Aa}^{*}$ & $17,40 \pm 0,16 \mathrm{Bb}$ & $22,50 \pm 0,13 \mathrm{Ac}$ & $29,50 \pm 0,26 \mathrm{Bd}$ & $34,10 \pm 0,16 \mathrm{Be}$ \\
CG 647 & $4,40 \pm 0,04 \mathrm{Aa}$ & $20,00 \pm 0,05 \mathrm{Ab}$ & $25,00 \pm 0,02 \mathrm{Ac}$ & $34,70 \pm 0,15 \mathrm{Ad}$ & $39,50 \pm 0,10 \mathrm{Ae}$ \\
CG 648 & $6,00 \pm 0,07 \mathrm{Aa}$ & $21,60 \pm 1,43 \mathrm{Ab}$ & $26,50 \pm 0,07 \mathrm{Ac}$ & $37,20 \pm 0,22 \mathrm{Ad}$ & $43,00 \pm 0,10 \mathrm{Ae}$ \\
\hline
\end{tabular}

Legenda: ${ }^{~=}$ Médias seguidas da mesma letra maiúscula na vertical e minúscula na horizontal não diferem entre si pelo teste de Tukey ao nível de $5 \%$ de significância. Fonte: Dados da pesquisa.

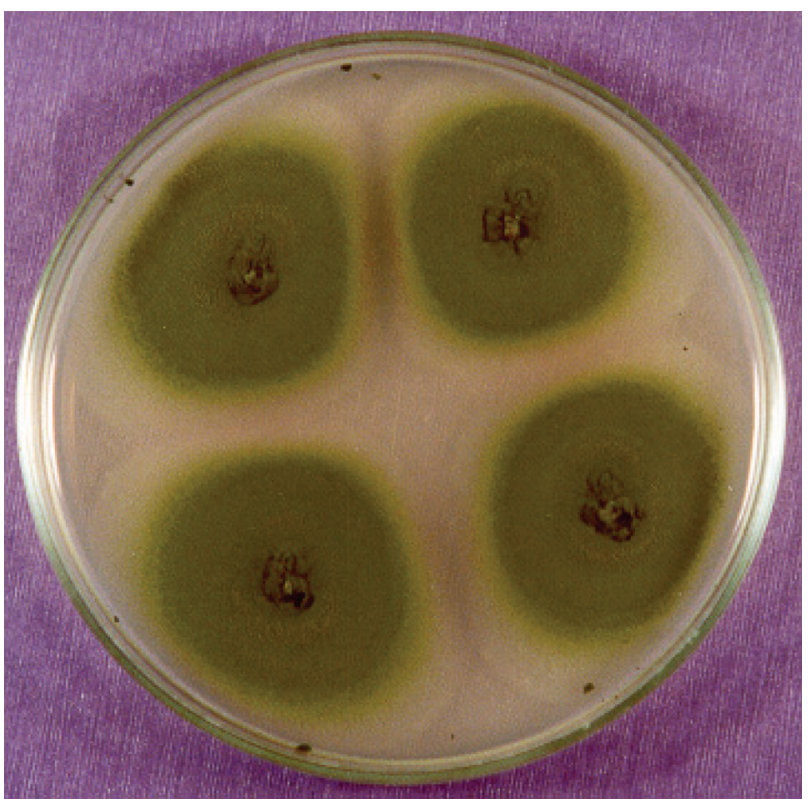

Figura 1 - Crescimento do fungo Metarhizium flavoviride var. flavoviride em meio BDA

CG-291 apresentou uma cinética de $\mathrm{y}=18,036 \ln (\mathrm{x})$ - 65,656; o fungo CG-647 com uma cinética de y = 17,269 $\ln (\mathrm{x})$ - 63,86; e o fungo CG-648 com a cinética de $\mathrm{y}=14,239 \ln (\mathrm{x})-51,341$.

Comparando os dados obtidos neste trabalho com os verificados por Tlecuitl (2009), verifica-se que o fungo $M$. anisopliae var. lepidiotum teve crescimento semelhante quando mantido em meio à base de arroz e farinha de aveia, acrescendo-se peptona a 1\%. Destaca-se que o comportamento semelhante está relacionado aos componentes do meio de cultura, à temperatura de crescimento e à oxigenação constante.

Os dados referentes ao crescimento do fungo Metarhizium flavoviride var. flavoviride também são

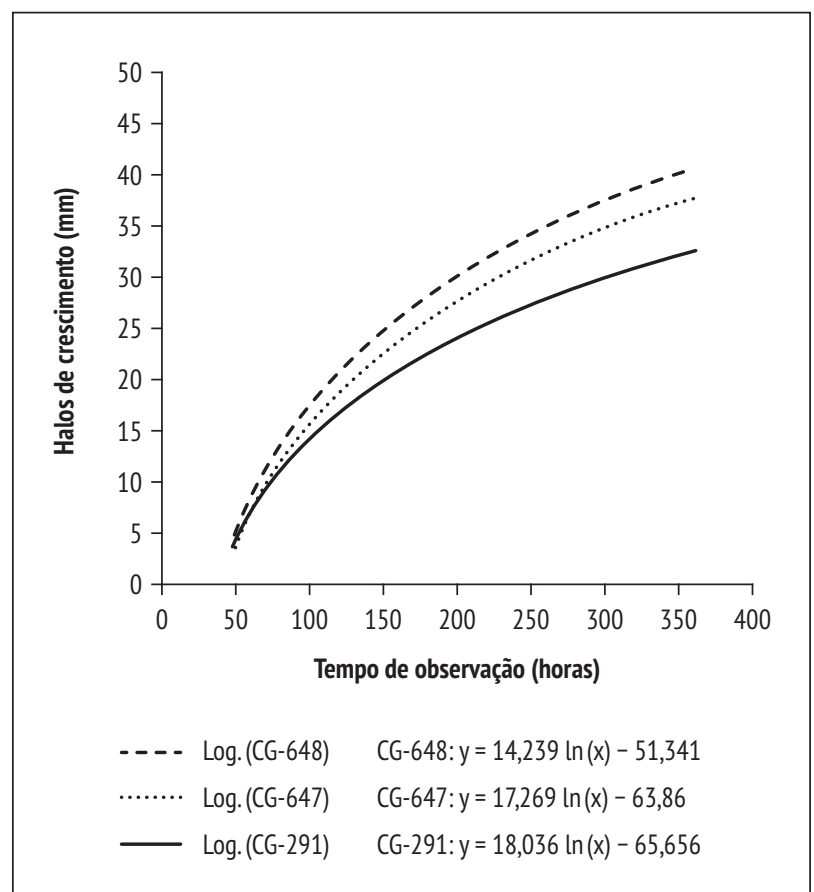

Figura 2 - Cinética de crescimento das três linhagens do fungo Metarhizium sp. em meio BDA sob condições controladas

Fonte: Dados da pesquisa.

semelhantes aos obtidos por Onofre et al. (2001) quando comparou o efeito de três meios de cultura: batata-dextrose-ágar (BDA), Czapek-ágar (CZP) e meio completo (MC); e três regimes de luminosidade: claro contínuo, alternância com luz do dia/ escuro e luz negra/escuro, sobre o crescimento e a esporulação desse mesmo fungo entomopatogênico. Os experimentos foram conduzidos nas mesmas condições e os resultados mostraram que o melhor crescimento ocorreu em meio BDA e MC. 
O isolado de M. anisopliae var. acridum apresentou padrão de crescimento similar aos outros fungos avaliados nas mesmas condições. Essa característica foi observada por Driver; Milner e Trueman (2000), que relatam que esse fungo tem bom comportamento em temperatura de $25{ }^{\circ} \mathrm{C}$, com crescimento micelial semelhante aos obtidos neste trabalho, formando halos de 42,09 $\mathrm{mm}$ sob as mesmas condições. A esporulação do fungo M. anisopliae var. acridum pode ser observada na Figura 3.

Os dados obtidos com as três linhagens do fungo Metarhizium, em relação ao comportamento inibitório dos fungicidas Nativo ${ }^{\circledR}$ e Bendazol ${ }^{\circledR}$, estão demonstrados pelas Tabelas 2 e 3, respectivamente.

Nas análises das Tabelas 2 e 3, percebe-se que em ambas ocorreu inibição do crescimento das três variedades, tanto na presença do fungicida Nativo ${ }^{\circledR}$ quanto na presença do fungicida Bendazol ${ }^{\circledR}$.

Na Tabela 2, verifica-se que o fungicida Nativo ${ }^{\circledR}$ inibiu totalmente o crescimento da linhagem CG-291 de Metarhizium anisopliae var. acridum, na concentração recomendada para o uso no campo, que corresponde a $2,5 \mathrm{~mL} / \mathrm{L}$. A partir dessa concentração, as diluições realizadas foram de 1,250; 650; 350; $150 ; 70 ; 40 \mu \mathrm{L} / \mathrm{L}$ e a partir de $40 \mu \mathrm{L} / \mathrm{L}$ houve crescimento fúngico, em que a medida do halo de crescimento foi de 4,00 $\pm 0,05 \mathrm{~mm}$.

Com os resultados obtidos e expressos nas Tabelas 2 e 3, pode-se verificar que, para a variedade Metarhizium anisopliae var. acridum, a concentração inibitória mínima (CIM) obtida com as concentrações avaliadas, para o fungicida Nativo ${ }^{\circledR}$ foi de $70 \mu \mathrm{L} / \mathrm{L}$ e para o Bendazol ${ }^{\circledR}, 170 \mu \mathrm{L} / \mathrm{L}$.

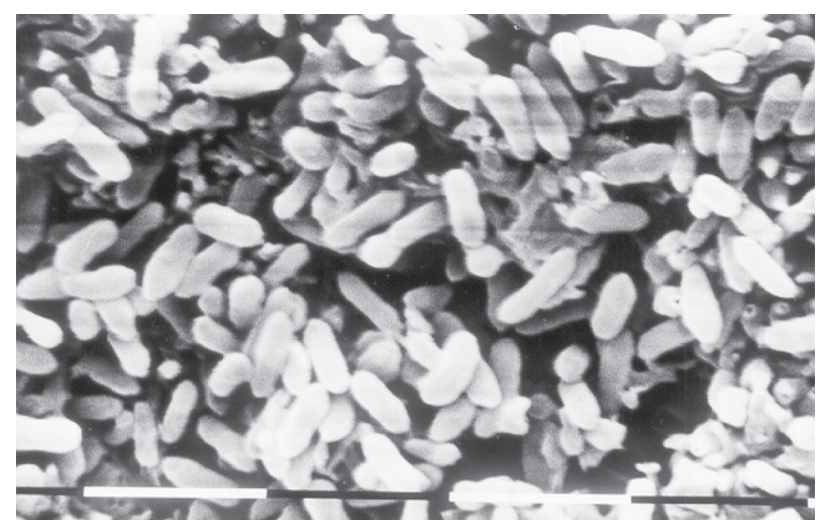

Figura 3 - Micrografia eletrônica de esporos do fungo $M$. anisopliae var. acridum em meio BDA

Fonte: ONOFRE, 2001
Deve-se também analisar que, apesar de ter ocorrido crescimento fúngico sob ação dos fungicidas Nativo $^{\circledR}{ }^{\circledR}$ Bendazol ${ }^{\circledR}$, os valores dos halos de crescimento obtidos são inferiores aos halos obtidos com o grupo controle $(34,10 \pm 0,16 \mathrm{~mm})$ nos mesmos tempos de observação. Assim, pode-se afirmar que existe forte inibição no crescimento fúngico diante dos fungicidas avaliados.

Diante do fungicida Nativo ${ }^{\circledR}$, o fungo Metarhizium flavoviride var. flavoviride linhagem CG 647 não se desenvolveu na concentração de $2,5 \mathrm{~mL} / \mathrm{L}$ estabelecida para campo, nem nas concentrações de 1,250; 650; 350 e $150 \mu \mathrm{L} / \mathrm{L}$ avaliadas. Já nas concentrações de 70 e $40 \mu \mathrm{L} / \mathrm{L}$ houve crescimento fúngico, sendo a medida do halo de 3,70 $\pm 0,06$ e 7,50 $\pm 0,06$, respectivamente.

0 fungo Metarhizium flavoviride var. flavoviride linhagem CG 647 não se desenvolveu na concentração estabelecida para campo $(1,4 \mathrm{~mL} / \mathrm{L})$ diante do fungicida Bendazol ${ }^{\circledR}$, nem nas concentrações de 750 e $350 \mu \mathrm{L} / \mathrm{L}$. Já nas concentrações de 170; 85; 43 e $22 \mu \mathrm{L} / \mathrm{L}$ avaliadas, o fungo apresentou crescimento, sendo este de 1,60 $\pm 0,06 ; 3,50 \pm 0,04 ; 8,50 \pm 0,06 \mathrm{e}$ $9,50 \pm 0,08$, respectivamente.

Após analise dos dados contidos nas Tabelas $2 \mathrm{e}$ 3 , verifica-se que, para o fungo Metarhizium flavoviride var. flavoviride linhagem CG 647, a concentração inibitória mínima (CIM) obtida com as concentrações avaliadas, para o fungicida Nativo ${ }^{\circledR}$ foi de $150 \mu \mathrm{L} / \mathrm{L}$ e para o Bendazol ${ }^{\circledR}, 350 \mu \mathrm{L} / \mathrm{L}$.

Apesar do desenvolvimento do fungo Metarhizium flavoviride var. flavoviride linhagem CG 647, diante dos fungicidas Bendazol ${ }^{\circledR}$ e Nativo ${ }^{\circledR}$, os valores dos halos de crescimento obtidos são inferiores aos halos obtidos com o grupo controle $(39,50 \pm 0,10 \mathrm{~mm})$ nos mesmos tempos de observação. Assim, pode-se observar que existe forte inibição no crescimento fúngico diante dos fungicidas avaliados.

Sob ação do fungicida Nativo ${ }^{\circledR}$, o fungo Metarhizium anisopliae var. lepidiotum linhagem CG 648 não apresentou crescimento na concentração estabelecida para campo $(2,5 \mathrm{~mL} / \mathrm{L})$, nem nas concentrações de 1,250; 650 e $350 \mu \mathrm{L} / \mathrm{L}$. Apesar disso, nas concentrações de 150, 70 e $40 \mu \mathrm{L} / \mathrm{L}$ houve desenvolvimento fúngico, sendo a medida dos halos de $1,50 \pm 0,04$; $2,50 \pm 0,04$ e 3,80 $\pm 0,02$, respectivamente.

Sob ação do fungicida Bendazol ${ }^{\circledR}$, o fungo Metarhizium anisopliae var. lepidiotum linhagem CG 648 não se desenvolveu na concentração de $1,4 \mathrm{~mL} / \mathrm{L}$ 
Tabela 2 - Características de crescimento das três linhagens do fungo Metarhizium em meio BDA sob condições controladas na presença do fungicida Nativo ${ }^{\circledR}-360$ horas de crescimento

\begin{tabular}{lccccc}
\hline & \multicolumn{5}{c}{ Tempo de observação (horas) / halos de crescimento em $\mathbf{~ m m}$} \\
\cline { 2 - 6 } Variedade & $\mathbf{2 , 5 ^ { * }}$ & $\mathbf{1 . 2 5 0 \#}$ & $\mathbf{1 5 0 \#}$ & $\mathbf{7 0 \#}$ & $\mathbf{4 0 \#}$ \\
\hline CG 291 & & $\mathrm{NC}$ & $\mathrm{NC}$ & $\mathrm{NC}$ & $4,00 \pm 0,05 \mathrm{~B}$ \\
CG 647 & $\mathrm{NC}$ & $\mathrm{NC}$ & $3,70 \pm 0,06 \mathrm{Aa}$ & $7,50 \pm 0,06 \mathrm{Ab}$ \\
CG 648 & $\mathrm{NC}$ & $\mathrm{NC}$ & $1,50 \pm 0,04 \mathrm{Aa}$ & $2,50 \pm 0,04 \mathrm{Aa}$ & $3,80 \pm 0,02 \mathrm{Ba}$ \\
\hline
\end{tabular}

Legenda: Médias seguidas da mesma letra maiúscula na vertical e minúscula na horizontal não diferem entre si pelo teste de Tukey ao nível de $5 \%$ de significância;

* = concentração recomendada para o campo em $\mathrm{mL} / \mathrm{L}$; \# = valores fornecidos em $\mu \mathrm{L} / \mathrm{L} ;{ }^{*} \mathrm{NC}=$ não houve crescimento.

Fonte: Dados da pesquisa.

Tabela 3 - Características de crescimento das três linhagens do fungo Metarhizium em meio BDA, sob condições controladas, na presença do fungicida Bendazol ${ }^{\circledR}-360$ horas de crescimento

\begin{tabular}{lccccc}
\hline & \multicolumn{5}{c}{ Tempo de observação (horas) / halos de crescimento em mm } \\
\cline { 2 - 6 } Variedade & $\mathbf{1 , 4 ^ { * }}$ & $170 \#$ & $\mathbf{8 5 \#}$ & $\mathbf{4 3 \#}$ & $\mathbf{2 2 \#}$ \\
\hline CG 291 & & $\mathrm{NC}$ & $2,20 \pm 0,06 \mathrm{Aa}$ & $3,50 \pm 0,10 \mathrm{Ba}$ & $8,20 \pm 0,04 \mathrm{Ab}$ \\
CG 647 & $\mathrm{NC}$ & $1,60 \pm 0,06 \mathrm{Aa}$ & $3,50 \pm 0,04 \mathrm{Ab}$ & $8,50 \pm 0,06 \mathrm{Ac}$ & $9,50 \pm 0,08 \mathrm{AC}$ \\
CG 648 & $\mathrm{NC}$ & $1,30 \pm 0,02 \mathrm{Aa}$ & $2,00 \pm 0,04 \mathrm{Aa}$ & $4,50 \pm 0,06 \mathrm{Bb}$ & $5,00 \pm 0,06 \mathrm{Bb}$ \\
\hline
\end{tabular}

Legenda: Médias seguidas da mesma letra maiúscula na vertical e minúscula na horizontal não diferem entre si pelo teste de Tukey ao nível de $5 \%$ de significância;

${ }^{*}$ = concentração recomendada para o campo em $\mathrm{mL} / \mathrm{L}$; \# = valores fornecidos em $\mu \mathrm{L} / \mathrm{L} ;{ }^{*}=\mathrm{NC}=$ não houve crescimento.

Fonte: Dados da pesquisa.

estabelecida para campo, nem nas concentrações de 750 e $350 \mu \mathrm{L} / \mathrm{L}$, mas cresceu nas concentrações de 170,85 , 43 e $22 \mu \mathrm{L} / \mathrm{L}$, com crescimento mostrando halos de $1,30 \pm 0,02 ; 2,00 \pm 0,04 ; 4,50 \pm 0,06$ e $5,00 \pm 0,06 \mathrm{~mm}$, respectivamente. Em análise desses dados, verifica-se que, para o fungo Metarhizium anisopliae var. lepidiotum, a concentração inibitória mínima (CIM) obtida com as concentrações avaliadas, para o fungicida Nativo ${ }^{\circledR}$ foi de $150 \mu \mathrm{L} / \mathrm{L}$ e de $350 \mu \mathrm{L} / \mathrm{L}$ para o Bendazol ${ }^{\circledR}$.

\section{Discussão}

Comparando os dados obtidos na presença dos fungicidas com o crescimento na ausência destes, percebe-se que os halos foram menores que os obtidos com o grupo controle, que foi de 43,00 $\pm 0,10$ $\mathrm{mm}$. Assim, pode-se afirmar que existe uma forte inibição no crescimento fúngico diante dos fungicidas avaliados.

De modo geral, analisando-se os dois fungicidas avaliados, diante de todas as linhagens analisadas, pode-se verificar que os crescimentos obtidos a partir da CIMs estabelecidas têm halos de crescimento muito inferiores aos halos obtidos com o grupo controle nos mesmos tempos de observação. Assim, pode-se afirmar que os fungicidas avaliados, diante das variedades de Metarhizium anisopliae, foram capazes de inibir o crescimento fúngico.

Segundo Ghini (2000), de acordo com o modo de ação de cada um dos princípios ativos avaliados verifica-se que as estrobilurinas possuem a ação translaminar, com atuação específica sobre o patógeno, e apresentam alto risco de resistência quando comparadas com os triazóis (Ciproconazol e Tebuconazole). As estrobilurinas interferem na respiração mitocondrial ao bloquear a transferência de 
elétrons pelo complexo citocromo bc1, inibindo, assim, a respiração celular do fungo.

Os triazóis afetam especificamente a divisão celular, pois apresentam atividade seletiva para a tubulina de fungos e ligam-se a essa proteína impedindo que ocorra a polimerização dos microtúbulos formadores do fuso mitótico (WHEELER et al., 1995).

A expressiva toxicidade dos fungicidas é um dos fatores que mais limita a utilização desse grupo de agente de controle químico, por isso, em uma estratégia de introdução conjunta dos fungicidas, com fungos entomopatogênicos (controle associado), sugere-se o uso de concentrações subletais (subconcentrações) para fungicidas incompatíveis, como $\mathrm{Nativo}^{\circledR} \mathrm{e}$ Bendazol ${ }^{\circledR}$, devendo essas concentrações ser iguais ou inferiores à concentração inibitória mínima, apresentando, assim, uma estratégia que permite reduzir a quantidade e o custo com o inimigo natural, a quantidade de resíduos químicos sobre os alimentos e a possibilidade de intoxicação dos trabalhadores rurais durante os processos de preparo da calda inseticida e sua aplicação.

De acordo com Alves, Moino e Almeida (1998) e Delai (2009), o controle biológico no Brasil é favorecido pelo clima e pela sua rica biodiversidade, resultando em uma enorme gama de antagonistas nativos de pragas, representados por seus parasitoides, predadores e patógenos. Além disso, os pequenos agricultores estariam mais propensos à adoção de métodos alternativos de controle de pragas e doenças, pois essa prática agregaria valor ao produto final, resultando em maior aceitação no mercado.

A grande variabilidade genética desses entomopatógenos pode ser considerada uma das suas principais vantagens no controle microbiano de insetos. Com técnicas apropriadas de bioensaios é possível selecionar isolados de fungos altamente virulentos, específicos ou não, com características adequadas, para serem utilizados como inseticidas microbianos, visando ao controle de grande número de pragas das culturas econômicas (ALVES, 1998a).

A utilização de patógenos associados às subdosagens de inseticidas químicos é uma prática que tem sido usada experimentalmente. A finalidade do emprego do inseticida químico é provocar estresse no inseto para torná-lo mais sensível ao patógeno (ALVES, 1998b). Com os resultados obtidos, pode-se verificar que os agricultores poderiam utilizar práticas menos agressivas ao meio ambiente do que o uso intensivo de agrotóxicos e fertilizantes químicos ou dosagens menores destes.

\section{Conclusão}

Pode-se concluir que os fungicidas Nativo ${ }^{\circledR}$, composto de trifloxistrobina + tebuconazole, e Bendazol ${ }^{\circledR}$, composto pelo princípio ativo carbendazin, inibem o crescimento das linhagens de Metarhizium sp. avaliadas. Contudo, a preservação dos fungos como agentes microbianos de ocorrência natural é essencial para evitar ressurgência ou surtos de pragas. A compatibilidade elevada com agroquímicos também pode auxiliar, melhorando seu potencial como agente de controle, pois as substâncias sintéticas podem atuar como estressantes, facilitando a infecção por fungos e sua rápida proliferação. Assim, sugerese que estudos futuros sejam realizados em ambientes de campo, utilizando-se os fungicidas analisados, para que se analise as interações entre estes e os fungos entomopatogênicos Metarhizium anisopliae var. acridum, Metarhizium anisopliae var. lepediotum e Metarhizium flavoviride var. flavoviride, para comprovação dos resultados encontrados in vitro.

\section{Referências}

ALVES, S. B. Agentes entomopatogênicos no controle microbiano. In: ALVES, S. B. (Coord.). Controle microbiano de insetos. São Paulo: Manole, 1986. p. 73-126.

ALVES, S. B. (Coord.). Controle microbiano de insetos. 2. ed. Piracicaba: FEALQ, 1998a.

ALVES, S. B. Fungos entomopatogênicos. In: ALVES, S. B. (Coord.). Controle microbiano de insetos. 2. ed. Piracicaba: FEALQ, 1998b. p. 289-381.

ALVES, S. B.; MOINO Jr., A.; ALMEIDA, J. E. M. Produtos fitossanitários e entomopatógenos. In: ALVES, S. B. (Coord.). Controle microbiano de insetos. 2. ed. Piracicaba: FEALQ 1998. p. 217-238.

ATHAYDE, A. C. R. Patogenicidade de Beauveria bassiana, Metarhizium anisopliae e Metarhizium flavoviride sobre ovos, larvas e teleóginas de Boophilus microplus da região semi-árida. 2002. 138 f. Tese (Doutorado em Ciências Biológicas) - Universidade Federal de Pernambuco, Recife, 2002. 
DELAI, L. S. et. al. Controle biológico na teoria e na prática: a realidade dos pequenos agricultores da região de Cascavel-PR. In: SEMINÁRIO INTERNACIONAL EXPERIÊNCIAS DE AGENDA 21: OS DESAFIOS DO NOSSO TEMPO, 1., 2009, Ponta Grossa. Anais... Ponta Grossa: Universidade Estadual de Ponta Grossa, 2009.

DESTÉFANO, R. H. R. Detecção e identificação de Metarhizium anisopliae em larvas de Diatraea Saccharalis por primers específicos. 2003. 87 f. Tese (Doutorado em Agronomia) - Escola Superior de Agricultura Luiz de Queiroz, Piracicaba, 2003.

DRIVER, F.; MILNER, R. J.; TRUEMAN, J. W. H. A taxonomic revision of Metarhizium based on a phylogenetic analysis of rDNA sequence data. Mycological Research, v. 104, p. $134-150,2000$.

GHINI, R.; KIMATI, H. Resistência de fungos a fungicidas. Jaguariúna: Embrapa Meio Ambiente, 2000.

MALDANER, G. et al. Pragas iniciais em soja no Sul do Brasil. 2009. Disponível em: <http://www.clicrbs.com. br/>. Acesso em: 25 jul. 2010.

MENEZES, L. A. Controle biológico: na busca pela sustentabilidade da agricultura brasileira. Revista Brasileira Campo \& Negócios, v. 72, p. 20-26, 2006.

ONOFRE, S. B. et al. Controle biológico de pragas na agropecuária por meio de fungos entomopatogênicos. In: SERAFINI, L. A.; BARROS, N. M.; AZEVEDO, J. L. (Org.). Biotecnologia - avanços na agricultura e na agroindústria. Caxias do Sul: EDUCS, 2002. p. 295-328.

ONOFRE, S. B. Variabilidade genética em Metarhizium flavoviride e sua patogenicidade sobre Boophilus microplus. 2001. 127 f. Tese (Doutorado em Processos Biotecnológicos) - Universidade Federal do Paraná, Curitiba, 2001.
ONOFRE, S. B. et al. Growth and sporulation of Metarhizium flavoviride var. flavoviride on culture media and lighting regimes. Scientia Agricola, v. 58, n. 3, p. 613616, 2001

SECRETARIA DA AGRICULTURA E DO ABASTECIMENTO DO PARANÁ - SEAB. Bendazol. 2010a. Disponível em: <http://www.seab.pr.gov.br/arquivos>. Acesso em: 15 set. 2010.

SECRETARIA DA AGRICULTURA E DO ABASTECIMENTO DO PARANÁ - SEAB. Nativo. 2010b. Disponível em: <http:// www.seab.pr.gov.br/arquivos>. Acesso em: 15 set. 2010.

TLECUITL, B. et al. Medium selection and effect of higher oxygen concentration pulses on Metarhizium anisopliae var. lepidiotum conidial production and quality. Mycophatologia, v. 169 , n. 5 , p. $387-394,2009$.

WHEELER, I. E. et al. Using allele-specific oligonucleotide probes to characterize benzimidazole resistance in Rhynchosporium secalis. Pesticide Science, v. 43, n. 3, p. 201-209, 1995.
Recebido: 19/11/2010

Received: $11 / 19 / 2010$

Aprovado: 26/01/2011

Approved: 01/26/2011 